\title{
Foreign body aspiration - still a challenge in pediatrics?
}

\author{
Cristina Iolanda Vivisenco ${ }^{1,2}$, Andra Farcasi ${ }^{1}$, Adelaida Iorgulescu ${ }^{3}$, Ioana Adelina Mertic ${ }^{3}$ \\ ${ }^{1}$ Department of Toxicology, "Grigore Alexandrescu" Emergency Children's Hospital, \\ Bucharest, Romania \\ 2Department of Pediatrics, "Carol Davila" University of Medicine and Pharmacy, Bucharest, Romania \\ ${ }^{3}$ Department of ENT, "Grigore Alexandrescu" Emergency Children's Hospital, Bucharest, Romania
}

\begin{abstract}
Tracheobronchial foreign body aspiration in children represents a diagnostic and therapeutic emergency in the practice of pediatricians and ENT specialists. Delaying the diagnostic and, implicitly, the bronchoscopic extraction of the foreign body can lead to complications, some even with vital prognosis. In this paper, the authors present from their personal experience two pediatric cases of tracheobronchial foreign bodies diagnosed and treated at different time intervals after the moment of aspiration.
\end{abstract}

Keywords: tracheobronchial foreign bodies, bronchoscopy, child

\section{INTRODUCTION}

Tracheobronchial foreign body aspiration in children is nowadays still a frequent condition encountered in the pediatric and ENT services, characterized by a wide spectrum of presentations, from minimal symptoms that can go unnoticed, to various degrees of respiratory failure and even death by suffocation (1).

Currently, in Romania there is no national database of the cases of tracheobronchial foreign body aspiration. The data published in the literature comes from research of national centers on limited groups of patients. In the United States, around 2000 pediatric cases with this diagnosis are estimated annually. The rate of in-hospital mortality associated with these cases is $1.8 \%$ and cerebral anoxic injury is reported in $2.2 \%$ of cases (2).

Most cases of tracheobronchial foreign body aspiration are reported in the first 3 years of life, with a maximum incidence in the age group of 1-2 years and a clear predominance of male gender (3). There are a number of factors favoring aspiration such as the age-specific tendency to explore the environment by introducing any object in the mouth, the small diameter of the airways that predisposes to obstruction and the lack of molars that prevent full chewing of foods (4).

In this paper we present two cases of tracheobronchial foreign body aspiration in toddlers, different by clinical and anamnestic features, diagnosed and treated together by the pediatrician and ENT specialist.

\section{Case 1}

2 years and 2 months old female patient was hospitalized in our department for fever, mixed dyspnea and foreign body aspiration suspicion. Her past medical history revealed that the patient is known from infancy with airway hyperresponsiveness in infectious context, being exposed to the respiratory tract infections of her older sister, who is frequenting the kindergarten. Her family history is insignificant. 
The onset of the current symptoms was 24 hours prior to admission with nasal obstruction and fever, for which she received symptomatic treatment. 15 hours before admission, while the child was eating peanuts, she had a sudden access of spastic cough followed by dyspnea. The physical examination upon admission revealed a child with influenced general state, with fever (38.8 degrees $\mathrm{C}$ ) and tachycardia (heart rate $=150$ beats per minute), with a running nose and an intense hyperemic pharynx. The pulmonary examination revealed spastic cough, mixed dyspnea, wheezing, polypnea (respiratory rate $=40$ respirations per minute). The breath sounds were bilaterally present, diminished at the base of the right hemithorax.

Emergency laboratory tests revealed leukocytosis $(19,000 / \mathrm{mmc})$ with neutrophilia $(74 \%)$ and a biological inflammatory syndrome (C reactive protein $=10.9 \mathrm{mg} / \mathrm{dl})$. The chest $\mathrm{X}$-ray showed increased bilateral pulmonary interstitial markings and hyperlucency in the right cardiac-diaphragmatic angle (Figure 1). By correlating the radiologic images with the pulmonary stethacoustic and the suggestive history for a possible aspiration syndrome, we decided to perform emergency bronchoscopy under general anesthesia. Two large fragments of peanut were extracted from the right primitive bronchus (Figure 2).

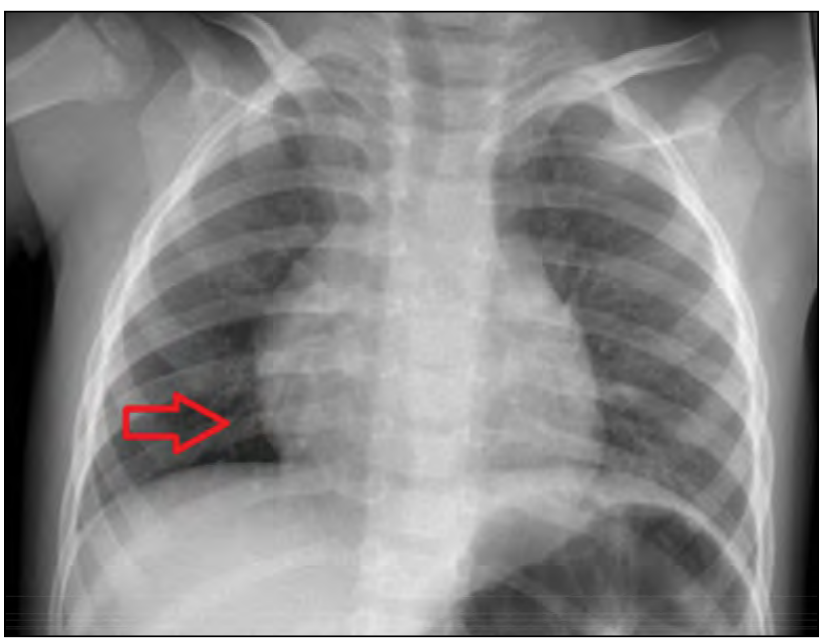

FIGURE 1. Slight pulmonary hyperlucency in the right cardiac-diaphragmatic angle

The patient received systemic antibiotherapy, inhaled corticotherapy and symptomatic medication. After the extraction of the foreign body, the clinical and paraclinical evolution was rapidly favorable. After the bronchoscopy, the child remained afebrile, without respiratory functional syndrome. The spastic cough persisted for 7 days and the physical examination revealed bronchial rales and a slightly prolonged expiration, which persisted for 48 hours. Because of these symptoms, we repeated the chest X-ray 5 days after the bronchoscopy. This showed the persistence of the bilateral pulmonary interstitial accentuation, without any ventilation disturbance. We repeated the laboratory tests, which revealed a normal leukocyte count and no inflammatory syndrome. We considered the persistence of the respiratory symptoms over several days as being caused by the overlap of foreign body aspiration with an acute respiratory tract infection in a child with a past medical history of airway hyperresponsiveness.

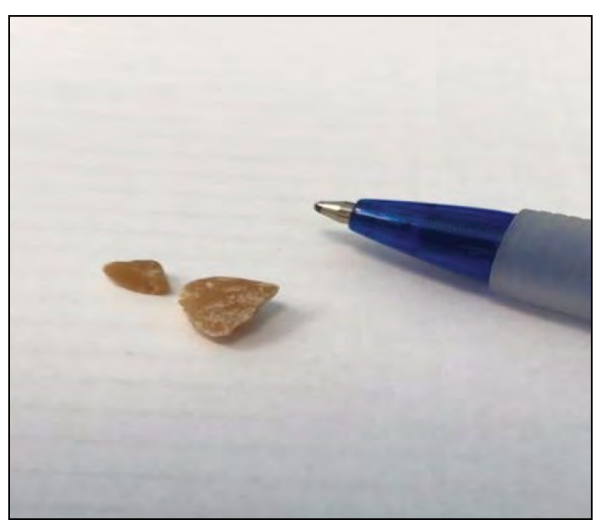

FIGURE 2. Fragments of peanut extracted with a rigid bronchoscope from the right primitive bronchus

The patient was discharged after 5 days and did not show any complications due to the foreign body aspiration during the 12 months of follow-up.

\section{Case 2}

3 years old female patient was hospitalized in our department for quasi permanent spastic cough and persistent wheezing. The family history revealed maternal allergic rhinitis and the patient past medical history showed repeated respiratory viral infections.

The onset of symptoms was one month prior to admission with dry cough, wheezing and fever (38 degrees C). She was hospitalized for two days in another medical unit with the diagnosis of laryngotracheobronchitis and she received treatment with oral antibiotics, inhaled bronchodilators and anti-inflammatory drugs. Because the symptoms persisted, she did two more consultations (pediatrics and pediatric pulmonology) and the diagnosis of virus-induced persistent wheezing was established. She received treatment for one month with oral leukotriene inhibitor and inhaled bronchodilators and anti-inflammatory drugs.

The physical examination upon admission revealed a child with influenced general state, afe- 
brile, with a suffering face. The pulmonary examination revealed quasi permanent, exhaustive, spastic cough, wheezing and, at auscultation, the breath sounds were diminished on the left hemithorax, at the base, with bronchial rales at the same site.

Analyzing the initial chest X-ray, which was taken at the onset of the symptomatology, one month earlier, we observed hyperlucency of the left hemithorax and mediastinal shift to the contralateral side (Figure 3a). The initial interpretation of the chest X-ray reported opacification of the right hemithorax, which probably led to the initial wrong interpretation as an acute lower respiratory tract infection. We repeated the chest X-ray upon admission in our clinic and we observed the persistence of the hyperlucency of the left hemithorax, less evident compared to the initial image, the aspect being attenuated by the overlap of a bilateral pulmonary interstitial accentuation (Figure 3b). Given the suggestive radiologic findings for foreign body aspiration, the medical history was resumed and the parents reported that the child "chocked with a peanut" on the day the symptoms started, claiming that they also mentioned this aspect at previous presentations. Taken into account the suggestive history and radiological findings, bronchoscopy was performed under general anesthesia. Two fragments of old peanut, encapsulated in granulation tissue, were extracted from the left primitive bronchus (Figure 4).

The patient received treatment with systemic antibiotherapy and corticotherapy. After the extrac- tion of the foreign body, the evolution was rapidly favorable with the complete disappearance of cough. At physical examination we noticed rare bronchial rales in the upper left hemithorax during the first 24 hours.

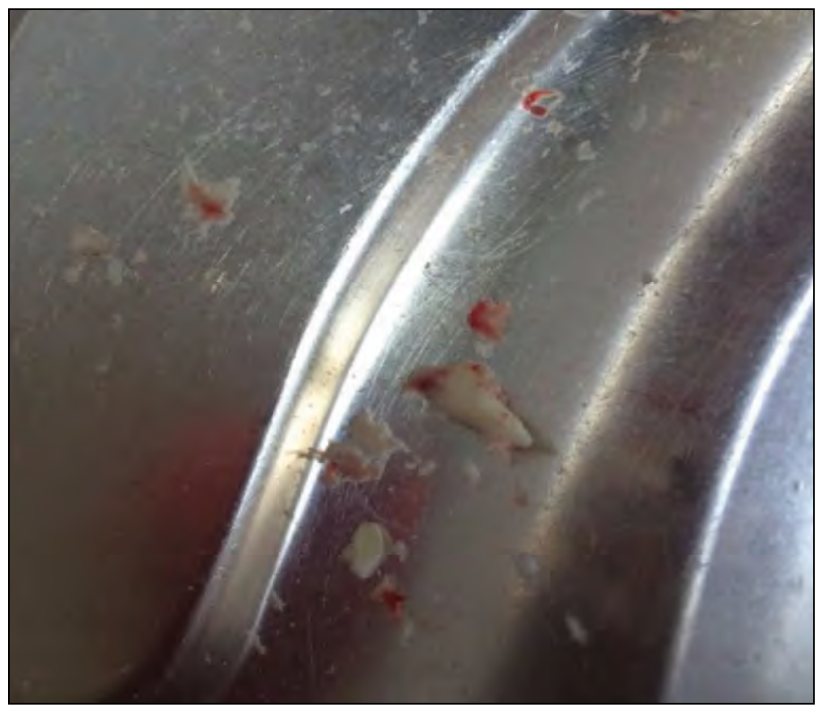

FIGURE 4. Old peanut fragments encapsulated in granulation tissue, extracted by bronchoscopy from the left primitive bronchus

The patient was discharged after 3 days, with recommendations of treatment with oral antibiotic and inhaled corticotherapy at home. The child has been followed-up for 24 months. In the first year, she had two episodes of acute lower respiratory tract infections, with the stethacoustic and radiological findings located in the left hemithorax. In

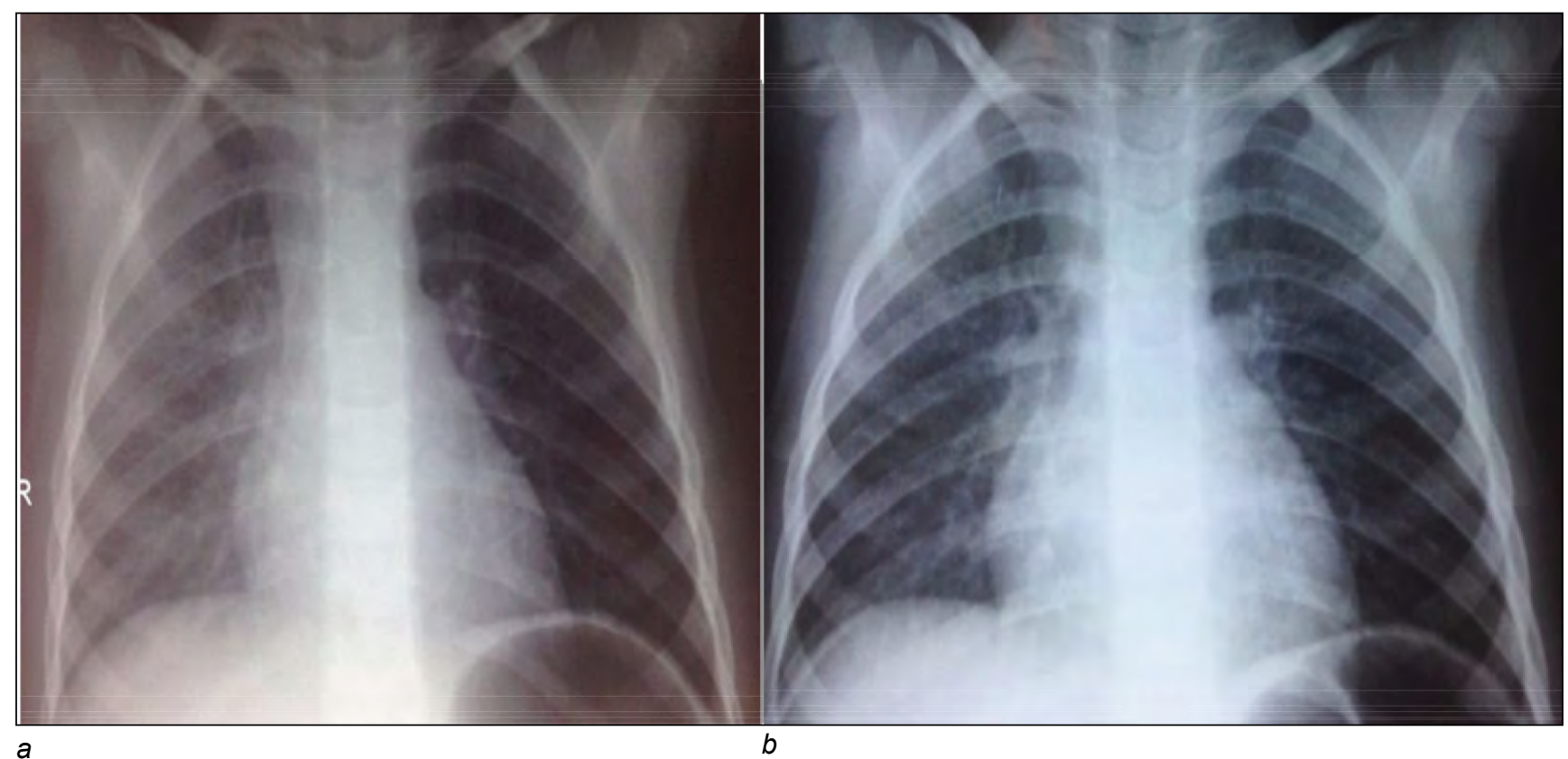

FIGURE 3 a. Chest X-ray performed at the time of foreign body aspiration. $b$. Chest X-ray performed one month after symptomatology onset 
the second year, she did not have any similar episodes. We have interpreted the recurrent infections as being favored by the structural changes in the respiratory tract, caused by the delayed diagnostic of foreign body aspiration.

\section{DISCUSSION}

In both cases we presented, tracheobronchial foreign bodies were of organic, vegetal nature. This is the most common type in medical practice, as is showed in Mallick's study of 158 children admitted for foreign body aspiration suspicion, $80 \%$ of cases being a vegetal foreign body (peanuts or melon seeds) (5). Similarly, Ciftci analyzed 663 pediatric cases, identifying organic foreign bodies in $75 \%$ of cases (sunflower seeds, peanuts, hazelnuts) (6).

Well guided anamnesis is essential in this condition, the history of penetration syndrome (sudden access of cough, suffocation, cyanosis) being the key element that guides the positive diagnosis. A study by Fontoba et al. on 100 patients showed that the presence of the penetration syndrome is the clinical parameter with the highest sensitivity $(97 \%)$ and specificity (63\%). Compared to this, the other symptoms and the radiological aspect, although having high sensitivity $(88 \%$ and $85 \%$, respectively), have low specificity (9\%) (7). There are many cases where the absence of witnesses at the time of the event does not allow the anamnestic identification of the penetration syndrome, which may lead to a delay in establishing the diagnostic. In both reported cases, the penetration syndrome was present, except that in the second case, the initial respiratory symptoms were not correctly interpreted, which led to a delay in establishing the diagnostic. A study by Steen and Zimmermann, on 94 pediatric cases of tracheobronchial foreign bodies, mentions that $24 \%$ of the patients were initially treated for another diagnosis and in $30 \%$ of cases the children were hospitalized more than 3 days after the moment of aspiration (8). Thus, foreign body aspiration in children can be erroneously diagnosed as an exacerbation of asthma, croup or recurrent wheezing (9). This initial wrong diagnostic situation was also found in our second case, which was treated for virus-induced persistent wheezing.

Foreign body aspiration can turn into a severe condition if it remains undiagnosed for a longer period of time. Delayed diagnosis and, implicitly, appropriate treatment can lead to complications such as pneumonia, recurrent infections, pulmonary atelectasis or bronchiectasis. A study by Karakoc et al. including 174 children diagnosed with foreign body aspiration revealed a $60 \%$ risk of complications occurring among those diagnosed more than 30 days after the aspiration of the foreign body. In $25 \%$ of cases diagnosed more than 30 days after aspiration, bronchiectasis was a major complication, 3 of the patients eventually requiring lobectomy (10). Similar results have been highlighted by Shizerman, who demonstrated that in 136 cases of foreign body aspiration, there has been a double occurrence of complications in patients who arrived at the hospital more than 2 days after the aspiration. Also, there was a double occurrence of complications in patients who performed bronchoscopy more than 24 hours after arriving at the hospital, compared with those who performed bronchoscopy in the first 24 hours (11). In the second case we presented, the delay in diagnosis led to recurrent pulmonary infections in the same area where the foreign body was located.

At the time of the physical examination, the child can be asymptomatic or may have one of the many clinical pictures which the presence of a tracheobronchial foreign body involves. The clinical picture may consist of cough, wheezing, stridor, respiratory functional syndrome, hyper-resonance or dullness at percussion of the lungs, decrease of vesicular breath sounds, coarse crackles and/or wheezes or tubular breath sounds (12). The chest X-ray may help establishing the diagnosis when it shows atelectasis or areas of emphysema with or without mediastinal shift to the contralateral side. In addition, it may highlight complications such as pneumonia with or without pleural effusion, bronchopneumonia, pulmonary abscess, pneumothorax or pneumomediastinum. A normal radiological aspect or one which is not suggestive for foreign body aspiration does not exclude the diagnosis. Zerella et al. studied 265 cases of proven foreign body aspiration in children, of which 110 cases $(41.5 \%)$ had normal chest X-rays (13).

Performing bronchoscopy under general anesthesia remains the best method of diagnosis and treatment. Examination of the tracheobronchial tree with a rigid bronchoscope should be performed in all cases of foreign body aspiration suspicion in children (14). The complications of bronchoscopy, although rare, may be severe: pneumothorax, hemorrhage, cardiopulmonary arrest (12). In Ciftci's study, the bronchoscopic removal of foreign bodies was successfully performed in $99 \%$ of cases. A second and a third bronchoscopy was required in a small number of cases $(3.2 \%$ and $0.8 \%$ respectively) (6). 
Investigation by computed tomography is a diagnostic option when the suspicion of tracheobronchial foreign body is high, despite a negative bronchoscopy or in cases where a residual foreign body is suspected after bronchoscopic extraction $(12,15)$.

\section{CONCLUSION}

A high clinical suspicion and a well guided anamnesis are essential in the early diagnosis of tracheobronchial foreign bodies. Delaying the diagno- sis will lead to inappropriate treatments, repeated hospitalizations or prolonged hospitalization and even immediate and distant complications. Bronchoscopy remains the method of choice for diagnosing and treating tracheobronchial foreign bodies.

\section{Acknowledgement}

The first two authors contributed equally.

Conflict of interest: none declared Financial support: none declared

\section{REFERENCES}

1. Naragund Al, Mudhol RS, Harugop AS. Tracheo-bronchial foreign body aspiration in children: A one year descriptive study. Indian J Otolaryngol Head Neck Surg. 2011; 66(Suppl 1): p. 180-5.

2. Kim IA, Shapiro N, Bhattacharyya N. The national cost burden of bronchial foreign body aspiration in children. Laryngoscope. 2015; 125(5): p. 1221-4.

3. Eren S, Balci AE, Dikici B. Foreign body aspiration in children: Experience of 1160 cases. Ann Trop Paediatr. 2003; 23(1): p. 31-7.

4. Committee on Injury, Violence, and Poison Prevention. Prevention of choking among children. Pediatrics. 2010; 125(3): p. 601-7.

5. Mallick MS. Tracheobronchial foreign body aspiration in children: A continuing diagnostic challenge. Afr J Paediatr Surg. 2014; 11(3): p. 225-8.

6. Ciftci AO, Bingöl-Koloğlu M, Senocak ME. Bronchoscopy for evaluation of foreign body aspiration in children. J Pediatr Surg. 2003; 38(8): p. 1170-6.

7. Fontoba JEB, Gutierrez C, Lluna J. Bronchial foreign body: Should bronchoscopy be performed in all patients with a choking crisis? Pediatr Surg Int. 1997; 12(2/3): p. 118-20.

8. Steen $\mathrm{KH}$, Zimmermann T. Tracheobronchial aspiration of foreign bodies in children: a study of 94 cases. Laryngoscope. 1990; 100(5): p. 525-30.

9. Mantor PC, Tuggle DW, Tunell WP. An appropriate negative bronchoscopy rate in suspected foreign body aspiration. Am J Surg. 1989; 158(6): p. 622-4.

10. Karakoç F, Karadağ B, Akbenlioğlu C. Foreign body aspiration: What is the outcome? Pediatr Pulmonol. 2002; 34(1): p. 30-6.

11. Shlizerman L, Mazzawi S, Rakover Y. Foreign body aspiration in children: The effects of delayed diagnosis. Am J Otolaryngol. 2010; 31(5): p. 320-4.

12. Hitter A, Hullo E, Durand C. Diagnostic value of various investigations in children with suspected foreign body aspiration: Review. Eur Ann Otorhinolaryngol Head Neck Dis. 2011; 128(5): p. 248-52.

13. Zerella JT, Dimler M, McGill LC. Foreign body aspiration in children: Value of radiography and complications of bronchoscopy. J Pediatr Surg. 1998; 33(11): p. 1651-4.

14. Acharya K. Rigid Bronchoscopy in Airway Foreign Bodies: Value of the Clinical and Radiological Signs. Int Arch Otorhinolaryngol. 2016; 20(3): p. 196-201.

15. Shin SM, Kim WS, Cheon JE. CT in Children with Suspected Residual Foreign Body in Airway after Bronchoscopy. AJR Am J Roentgenol. 2009; 192(6): p. 1744-51. 